\title{
Implementation of Combinatorial Algorithms using Optimization Techniques
}

\author{
Youssef Bassil \\ Researcher, LACSC - Lebanese Association for Computational Sciences Registered, Beirut, Lebanon
}

\begin{abstract}
How to cite this paper: Youssef Bassil "Implementation of Combinatorial Algorithms using Optimization Techniques" Published in International Journal of Trend in Scientific Research and Development (ijtsrd), ISSN: 24566470, Volume-3 | Issue-3, April 2019, pp.660-666, URL: http://www.ijtsrd.co $\mathrm{m} /$ papers/ijtsrd229 25.pdf

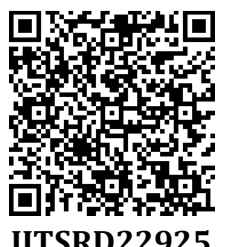

Copyright (C) 2019 by author(s) and International Journal of Trend in Scientific Research and Development Journal. This is an Open Access article distributed under the terms of the Creative Commons

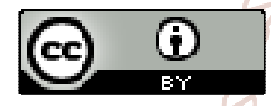
Attribution License (CC BY 4.0) (http://creativecommons.org/licenses/ by/4.0)

\section{ABSTRACT}

In theoretical computer science, combinatorial optimization problems are about finding an optimal item from a finite set of objects. Combinatorial optimization is the process of searching for maxima or minima of an unbiased function whose domain is a discrete and large configuration space. It often involves determining the way to efficiently allocate resources used to find solutions to mathematical problems. Applications for combinatorial optimization include determining the optimal way to deliver packages in logistics applications, determining taxis best route to reach a destination address, and determining the best allocation of jobs to people. Some common problems involving combinatorial optimizations are the Knapsack problem, the Job Assignment problem, and the Travelling Salesman problem. This paper proposes three new optimized algorithms for solving three combinatorial optimization problems namely the Knapsack problem, the Job Assignment problem, and the Traveling Salesman respectively. The Knapsack problem is about finding the most valuable subset of items that fit into the knapsack. The Job Assignment problem is about assigning a person to a job with the lowest total cost possible. The Traveling Salesman problem is about finding the shortest tour to a destination city through travelling a given set of cities. Each problem is to be tackled separately. First, the design is proposed, then the pseudo code is created along with analyzing its time complexity. Finally, the algorithm is implemented using a high-level programming language. As future work, the proposed algorithms are to be parallelized so that they can execute on multiprocessing environments making their execution time faster and more scalable.

KEYWORDS: Combinatorial Algorithms, Optimization Techniques, Knapsack, Job Assignment, Traveling Salesman

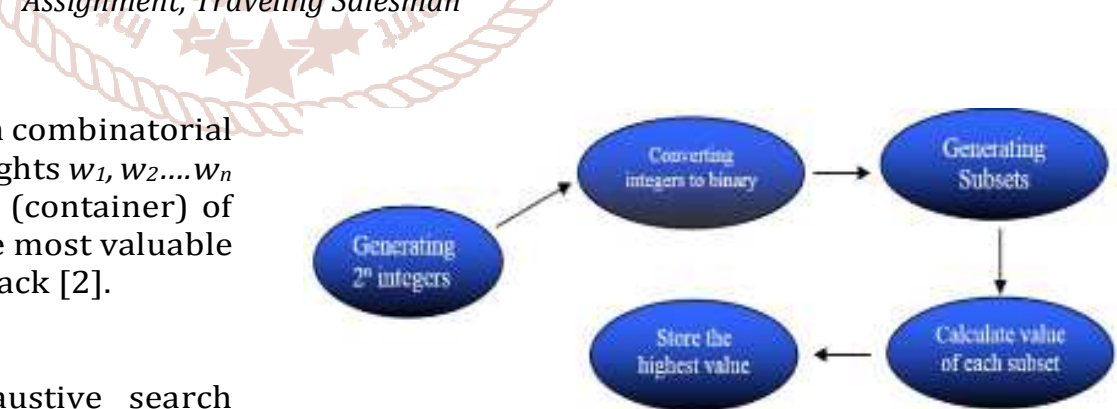

Figure 1: Process Flow for the Knapsack problem

C. Algorithm

//ALGORITHM Knapsack (itemsValue[n], items Weight[n])

// Knapsack Problem

// INPUT: itemsValue[n] , itemsWeight[n]

// OUTPUT: optimalSubset: array of integers

ITEMS_COUNT: integer constant that holds the \# of items

itemsValue[n]: array of integers that holds item Values

itemsWeight[n]: array of integers that holds item Weights 
bitString[ITEMS_COUNT]: array of flags that holds a particular subset

optimalSubset[ITEMS_COUNT]: array of flags that holds the subset of items with highest total value

knapsackCapacity : integer that holds the Capacity of the Knapsack

optimalValue: integer that holds the highest Value calculated after each subset

sumValues: integer that holds the sum of all items values for a given subset

sumWeights: integer that holds the sum of all items weights for a given subset

\section{BEGIN}

optimalValue $\leftarrow 0$

// Step1: Generates integer numbers FOR i <- 0 TO Pow(2,ITEMS_COUNT) DO \{

// Step2: Convert integer Numbers to binary numbers

// Step3: Generating Subsets

$\mathrm{j}<-0$

WHILE $\mathrm{i}<>0$

\{

bitString $[\mathrm{j}] \leftarrow \mathrm{i}$ MOD 2

$\mathrm{i} \leftarrow \mathrm{i} / 2$

\}

// Step4: Calculate the Item values corresponding to each subset sumValues $<-0$ sumWeights $<-0$

FOR $\mathrm{k}<-0$ TO ITEMS_COUNT DO

\{

// Replaces TRUE flag with its corresponding Item value

IF bitString $[\mathrm{k}]=$ TRUE THEN

\{

sumValues <- sumValues + itemsValue $[\mathrm{k}]$ sumWeights $<-$ sumWeights +

\} itemsWeight[k]

$\mathrm{k} \leftarrow \mathrm{k}+1$

\}

// Step5: Store the highest value with its corresponding subset

IF (sumWeights <= knapsackCapacity AND sumValues $>$ optimalValue)

\section{THEN}

\{

optimalValue <- sumValues

FOR $\mathrm{p} \leftarrow 0$ TO ITEMS_COUNT DO

\{

optimalSubset[p] <- bitString[p]

\} $\mathrm{p}<-\mathrm{p}+1$

$\mathrm{i} \leftarrow \mathrm{i}+1$

\} // end of step1 FOR LOOP

// Step6: Return the Subset that has highest Items value

RETURN optimalSubset

END

\section{Analysis}

The proposed algorithm can find the optimal subset of items with their corresponding optimal value while falling under the below efficiency class:

Knapsack $(a[n], b[n]) € 0 \quad n^{2} \quad\left(n^{2}>n\right)$

Knapsack $(a[n], b[n]) € \begin{array}{llll}\Omega & 1 & (1<n)\end{array}$

Knapsack $(a[n], b[n]) € \Phi n \quad(n=n)$

Performance wise, it requires about 9 milliseconds to handle the problem with 50 items.

\section{E. Implementation}

Figure 2 depicts the screenshot of the program that implements the Knapsack problem using C\#.NET [3].

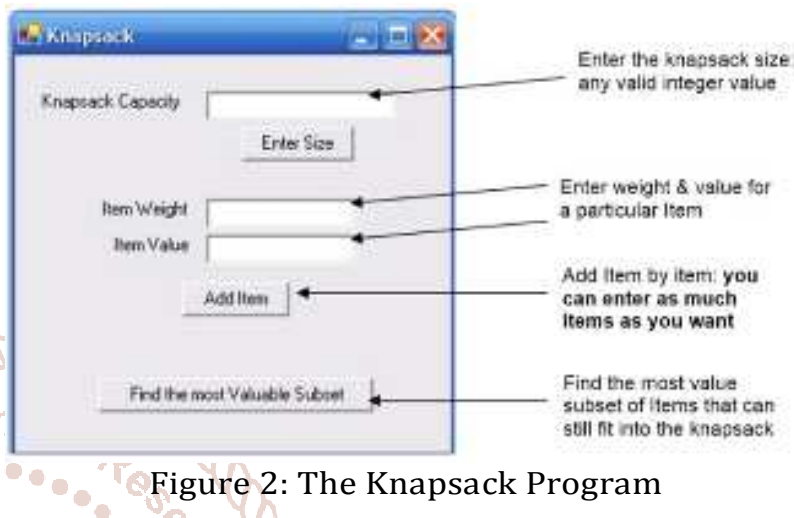

II. JOB ASSIGNMENT PROBLEM

The assignment problem is a fundamental combinatorial optimization problem [4]. Given $n$ people who need to be assigned to $n$ jobs, one person per job. The cost of ith person is assigned to jth job is stored in table[i][j]. The problem is to find an assignment with the lowest total cost [5].

\section{A. Proposed Solution}

Developing an algorithms based on the brute force techinque which tests and evaluates all possible objects combinations involved in the problem and performs appropriate calculations. The algorithm uses a one-dimentational array to store permutations and a two-dimentinal array to store Person/Job cost

\section{B. Design}

Figure 3 shows the process flow diagram of the Job Assignment problem design

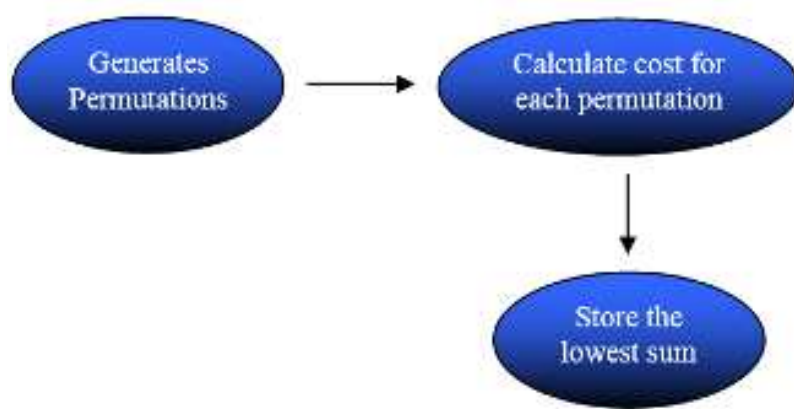

Figure 3: Process Flow for the Job Assignment problem

\section{Algorithm}

// ALGORITHM Assignment (table[n][n] , COUNTER) // Person/Job Assignment Problem // INPUT: table[n][n] , COUNTER 
// OUTPUT: optimalList : array of integers

table $[n][n]: 2 \mathrm{D}$ integer array that Stores all costs entered by the user

COUNTER: integer that holds the \# of persons(or the \# of jobs)

list[COUNTER]: array of integers that holds permutation

pointers[COUNTER]: array of integers that holds present direction of each permutation

increasingPtr[COUNTER]: array of integers that holds left to right arrows $->->->$....

decreasingPtr[COUNTER]: array of integers that holds right to left arrows $<-<-<-\ldots .$.

optimalSum: integer that holds the lower cost per person/job assignment

optimalList [COUNTER]: array of integers that holds the permutation with the lower cost

mobile: integer that holds the mobile element mobileIndex: integer that holds the index of the mobile element

flag: boolean variable that indicates if a mobile exists or not

temp: integer used FOR swapping purposes

sum: integer that holds the cost of a particular permutation instance

\section{BEGIN}

optimalSum $\leftarrow$ INFINITY

//Fill array lists with 12345 6....(depending on variable COUNTER) FOR $\mathrm{i} \leftarrow 0$ TO COUNTER DO \{

$\operatorname{list}[\mathrm{i}] \leftarrow \mathrm{i}+1$

$\mathrm{i} \leftarrow \mathrm{i}+1$ \}

//Initialize pointers <- <- <- .... FOR $\mathrm{i} \leftarrow$ COUNTER-1 TO 0 DO \{ pointers $[\mathrm{i}] \leftarrow \mathrm{i}-1$ $\mathrm{i} \leftarrow \mathrm{i}+1$ \}

//Initialize increasingPtr $->->->\ldots$. FOR $\mathrm{i} \leftarrow 0$ TO COUNTER DO

\{

increasingPtr $[\mathrm{i}] \leftarrow \mathrm{i}+1$

$\mathrm{i} \leftarrow \mathrm{i}+1$ \}

//Initialize decreasingPtr <- <- <- .... FOR $\mathrm{i} \leftarrow$ COUNTER-1 TO 0 DO

\{

decreasingPtr $[\mathrm{i}] \leftarrow \mathrm{i}-1$

$\mathrm{i} \leftarrow \mathrm{i}+1$

\}

// Johnson-Trotter ALGORTIHM

// Generates Permutations

FOR $\mathrm{i} \leftarrow 0$ TO fac(COUNTER)-1 DO

\{

//Calculate the cost for each permutation

instance

sum $\leftarrow 0$

FOR $\mathrm{j} \leftarrow 0$ TO COUNTER DO

\{

sum $\leftarrow$ sum +table $[\mathrm{j}$, list $[\mathrm{j}]-1]$

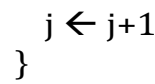

// Holds the lowest sum

IF sum < optimalSum THEN

\{

optimalSum $\leftarrow$ sum

FOR $\mathrm{k} \leftarrow 0$ TO COUNTER DO

\{

optimalList $[\mathrm{k}] \leftarrow$ list $[\mathrm{k}]$

$\mathrm{k} \leftarrow \mathrm{k}+1$

\}

\}

mobile $\leftarrow 0$

mobileIndex $\leftarrow 0$

flag $\leftarrow$ false

//Step1 : Find the largest Mobile

FOR $\mathrm{i} \leftarrow 0$ TO COUNTER DO

\{

IF(pointers $[\mathrm{i}]<>1$ \& \& pointers $[\mathrm{i}]<>$ COUNTER

AND list [i] >mobile AND

list[pointers $[\mathrm{i}]]<\operatorname{list}[\mathrm{i}]$ )

THEN

\{

mobile <- list[i]

mobileIndex $<-\mathrm{i}$

flag <- TRUE

\}

$\mathrm{i} \leftarrow \mathrm{i}+1$

International \}ournal

// Step2: test whether a mobile was found

//Step3: Swap the mobile with the element that it points to

// Step4: Swap the pointers of mobile and the element that it points to

// Step5: Reverse Directions of all elements that are greater than mob

IF flag=TRUE THEN

\{

// Swap the mobile with the element that it points to

list[mobileIndex] $\leftarrow$ list[pointers[mobileIndex]] list[pointers[mobileIndex]] $\leftarrow$ mobile

IF(pointers[pointers[mobileIndex $]$ =mobileInde $\mathrm{x})$ THEN

\{

// Indicates the mobile is at the left side IF(pointers[mobileIndex] > mobileIndex) THEN

\{

// Swap the pointers of mobile and the element that it points to

Temp $\leftarrow$ pointers[pointers[mobileIndex]]

pointers[pointers[mobileIndex]] $\leftarrow$ pointers [mobileIndex]+1

\} pointers [mobileIndex $] \leftarrow$ temp-1

ELSE // Indicates the mobile is at the right side

\{

// Swap the pointers of mobile and the element that it points to 
International Journal of Trend in Scientific Research and Development (IJTSRD) @ www.ijtsrd.com eISSN: 2456-6470

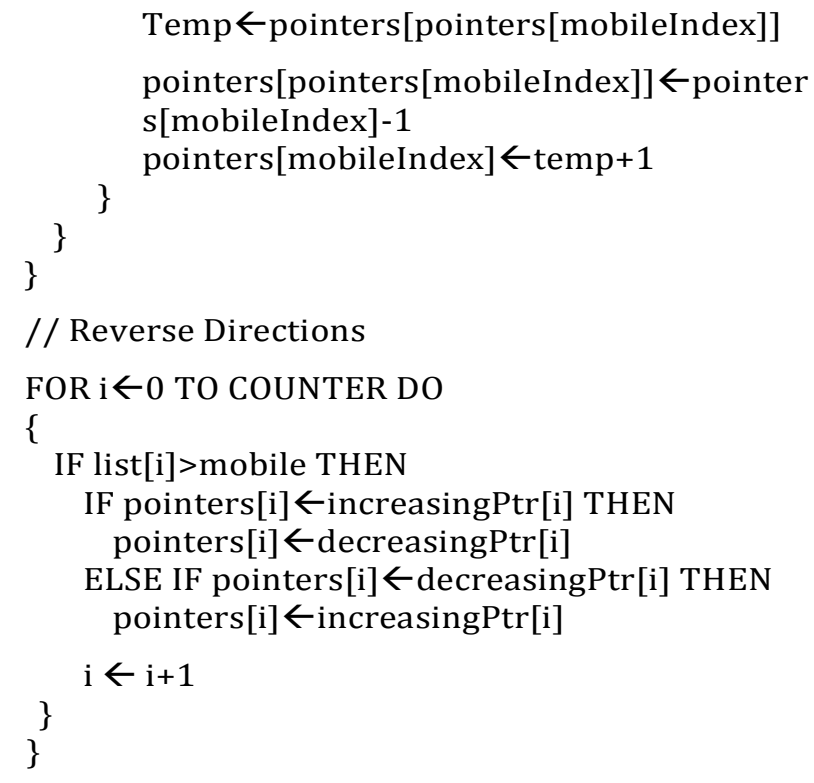

// Reverse Directions

//Calculate the cost FOR the last permutation instance sum $\leftarrow 0$

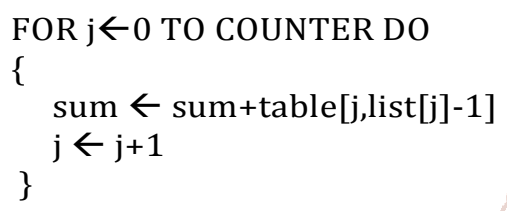

// Holds the lowest sum IF sum < optimalSum THEN \{

optimalSum $\leftarrow$ sum FOR $\mathrm{k} \leftarrow 0$ TO COUNTER DO \{ optimalList $[\mathrm{k}] \leftarrow$ list $[\mathrm{k}]$ $\mathrm{k} \leftarrow \mathrm{k}+1$$$
\text { ( }
$$

// optimal list should hold the less costly person/job assignment RETURN optimalList

\section{END}

\section{Analysis}

The proposed algorithm can find the optimal person/job assignment with its corresponding lowest cost. It is very practical even on large number of persons, however it exhausts processing time due to Johnson-trotter algorithm [6] whose order of growth is always exponential. The algorithm falls under the below efficiency class:

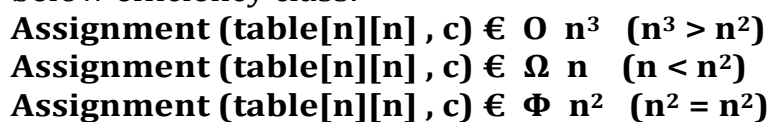

Performance wise, it requires 12 seconds to handle a problem with 100 jobs $100 !=9.33262154439441$ $52681699238856267 e+157$ permutations

\section{E. Implementation}

Figure 4 depicts the screenshot of the program that implements the Job Assignment problem using C\#.NET.

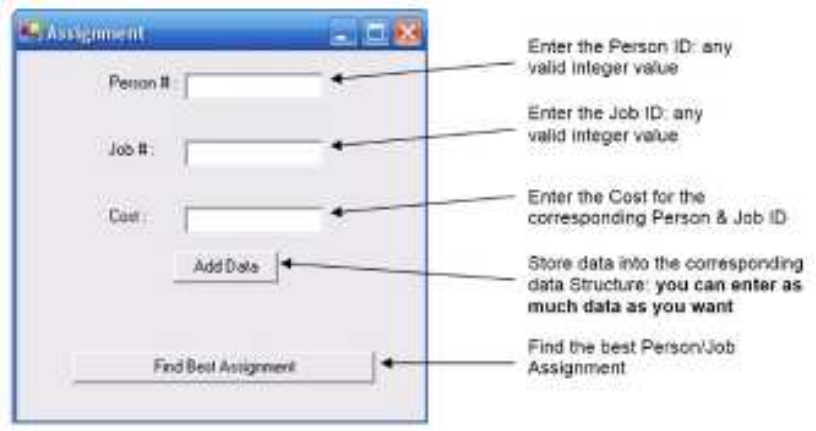

Figure 4: The Job Assignment Program

\section{TRAVELING SALESMAN PROBLEM}

The Traveling Salesman Problem is a classic algorithmic problem in the field of computer science that focuses on optimization [7]. The problem ask to find the shortest tour through a given set of $n$ cities or nodes that visits each city exactly once before returning to the city where it started [8].

\section{A. Proposed Solution}

Exaustive search technique is so far the most appropriate appraoch to solve this problem. It consists of generating all possible paths with their correponding lengths so eventually the shortest path can be identified. The algorithm uses a onedimentional array to store permutations, a onedimentional array to store distinct cities, and a twodimentional array to store from city, to city, and length variables.

B. Design

Figure 5 shows the process flow diagram for the Traveling Salesman problem design

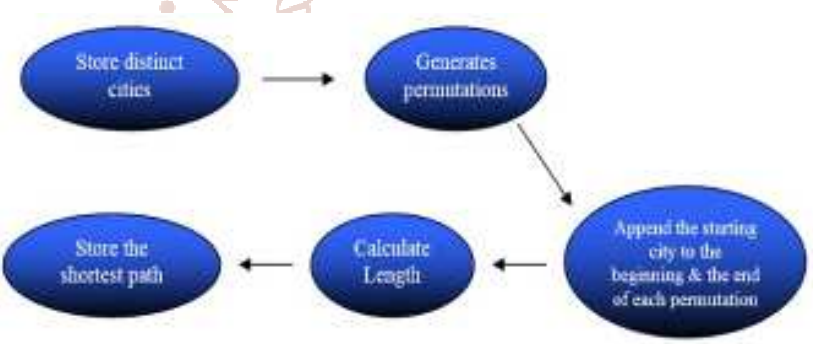

Figure 5: Process Flow for the Traveling Salesman problem

\section{Algorithm}

// ALGORITHM Salesman(table[n][3] , startCity) // Person/Job Assignment Problem // INPUT: table[n][n] , startCity // OUTPUT: optimalList : array of characters

cities [citiesCounter]: array of characters holds Distinct cities

newList [citiesCounter+1]: array of characters that holds: startcity+permutation+startcity

citiesCounter: integer holds \# of distinct cities startCity: Character holds the name of the start city table[n][3]: 2D integer array that Stores all routes with their corresponding length

list [citiesCounter-1]: array of characters that holds permutation

pointers[citiesCounter-1]: array of integers that holds present direction of each permutation 
increasingPtr[citiesCounter-1]: array of integers that holds left to right arrows -> -> ->

decreasingPtr[citiesCounter-1]: array of integers that holds right to left arrows <- <- <-

optimalSum: integer that holds the shortest path summation

optimalList [citiesCounter+1]: array of characters that holds the permutation with the shortest path mobile: integer that holds the mobile element mobileIndex: integer that holds the index of the mobile element

flag: boolean variable that indicates if a mobile exists or not

temp: integer used for swapping purposes

sum: integer that holds the cost of a particular permutation instance

\section{BEGIN}

//Step1: Recognize and store in array cities only the distinct cities

$\mathrm{i} \leftarrow 0$

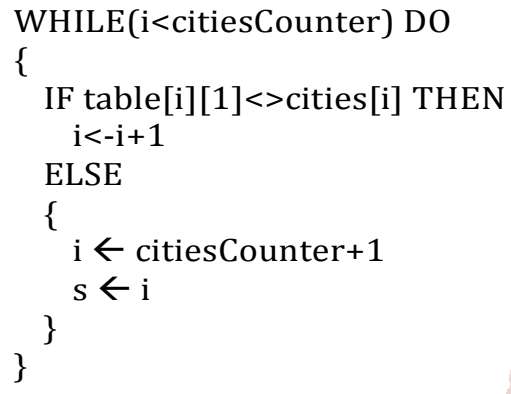

//Step2: create an array named list that contains all distinct cities

$\mathrm{k} \leftarrow 0$

FOR $\mathrm{i} \leftarrow 0$ TO citiesCounter DO

\{

IF cities [i] $<>$ startCity THEN

\{

list $[\mathrm{k}] \leftarrow \operatorname{cities}[\mathrm{i}]$

$\mathrm{k} \leftarrow \mathrm{k}+1$

\}

$\mathrm{i} \leftarrow \mathrm{i}+1$

\}

//Initialize pointers <- <-<- ....

FOR i $\leftarrow$ citiesCounter-1 TO 0 DO

\{

pointers $[\mathrm{i}] \leftarrow \mathrm{i}-1$

$\mathrm{i} \leftarrow \mathrm{i}+1$

\}

//Initialize increasingPtr -> -> -> ....

FOR $\mathrm{i} \leftarrow 0$ TO citiesCounter DO

\{

increasingPtr $[\mathrm{i}] \leftarrow \mathrm{i}+1$

$\mathrm{i} \leftarrow \mathrm{i}+1$

\}
//Initialize decreasingPtr <- <- <- ....

FOR i $\leftarrow$ citiesCounter- 1 TO 0 DO

\{

decreasingPtr $[\mathrm{i}] \leftarrow \mathrm{i}-1$

$\mathrm{i} \leftarrow \mathrm{i}+1$

\}

FOR $\mathrm{i} \leftarrow 0$ TO fac(citiesCounter)-1 DO

\{

// Step3 : Add the startcity at the beginning \& at the end

newList $[0] \leftarrow$ startCity

$\mathrm{k} \leftarrow 1$

FOR $s \leftarrow 0$ TO citiesCounter DO

\{

newList $[\mathrm{k}] \leftarrow$ list $[\mathrm{s}]$

$\mathrm{k} \leftarrow \mathrm{k}+1$

$\mathrm{s} \leftarrow \mathrm{s}+1$

\}

newList[citiesCounter]<-startCity

//Step4: Calculate Length

Sum $\leftarrow 0$

$\mathrm{i} \leftarrow 0$

$\mathrm{j} \leftarrow 0$

WHILE $\mathrm{i}<$ citiesCounter-1 AND $\mathrm{j}<\mathrm{n}-1$ DO

\{

IF(newList $[\mathrm{i}]=$ table $[\mathrm{j}, 0]$ AND

newList $[\mathrm{i}+1]=$ table $[\mathrm{j}, 1])$

THEN

\{

Sum $\leftarrow$ sum + table $[\mathrm{j}, 2]$

$\mathrm{i} \leftarrow \mathrm{i}+1$

$\mathrm{j} \leftarrow 0$

\}

ELSE $\mathrm{j} \leftarrow \mathrm{j}+1$

// store the shortest path

IF sum < optimalSum THEN

\{

optimalSum $\leftarrow$ sum

FOR $s \leftarrow 0$ TO $s<$ citiesCounter DO

\{

optimalList [s] $\leftarrow$ newList $[\mathrm{s}]$

\}

$\mathrm{s} \leftarrow \mathrm{s}+1$

\}

// Johnson-Trotter ALGORTIHM

// Step5: Generates Permutations

mobile $\leftarrow$ ' ' // small value

mobileIndex $\leftarrow 0$

flag $\leftarrow$ FALSE

// Step1 : Find the largest Mobile

FOR $\mathrm{i} \leftarrow 0$ TO citiesCounter DO

\{

IF (pointers $[\mathrm{i}]<>1$ AND

pointers[i]<>citiesCounter-1

AND list[i] >mobile AND

list[pointers[i]]<list [i])

THEN

\{

mobile $\leftarrow$ list $[\mathrm{i}]$

mobileIndex $\leftarrow$ i 


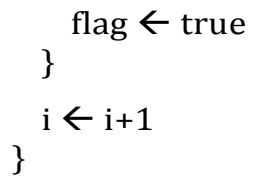

//Step2: test whether a mobile was found //Step3: Swap the mobile with the element that it points to

//Step4: Swap the pointers of mobile and the element that it points to

//Step5: Reverse Directions of all elements that are greater than mobile

IF flag=TRUE THEN

\{

// Swap the mobile with the element that it points to

list[mobileIndex] $\leftarrow$

list[pointers[mobileIndex]]

list[pointers[mobileIndex]] $\leftarrow$ mobile

IF(pointers[pointers[mobileIndex]]=mobileIn dex) THEN

\{

// Indicates the mobile is at the left side IF(pointers [mobileIndex] > mobileIndex) THEN

\{

// Swap the pointers of mobile and the element that it points to

Temp $\leftarrow$ pointers[pointers [mobileIndex]]

pointers[pointers[mobileIndex]] $\leftarrow$ pointer $\mathrm{s}[$ mobileIndex $]+1$

\} pointers [mobileIndex $] \leftarrow$ temp- 1

ELSE // Indicates the mobile is at the right side

\{

// Swap the pointers of mobile and the element that it points to

Temp $\leftarrow$ pointers[pointers[mobileIndex]]

pointers[pointers[mobileIndex] $\leftarrow$ pointer $\mathrm{s}$ [mobileIndex]-1 pointers $[$ mobileIndex $] \leftarrow$ temp +1 \}

\}

\}

\section{// Reverse Directions}

FOR $\mathrm{i} \leftarrow 0$ TO citiesCounter DO

\{

IF list [i] > mobile THEN

IF pointers[i] $\leftarrow$ increasingPtr[i] THEN

pointers $[\mathrm{i}] \leftarrow$ decreasingPtr $[\mathrm{i}]$

ELSE IF pointers $[\mathrm{i}] \leftarrow$ decreasingPtr[i] THEN pointers $[\mathrm{i}] \leftarrow$ increasing $\mathrm{Ptr}[\mathrm{i}]$

\}

$\mathrm{i} \leftarrow \mathrm{i}+1$

\}

RETURN optimalList

END

\section{Analysis}

The proposed algorithm can find the shortest path among many alternatives starting from a given city, passing through all the available cities only once to end at the same starting point. Even though it is based on Johnson-Trotter algorithm to generate permutations, the proposed algorithm is considered quite efficient due to the complexity of the original problem. Therefore to solve a complex problem such the traveling salesman problem, somehow you are going to lose some processing time. The algorithm falls under the below efficiency class:

Salesman (table[n][3], sCity) $€ O \quad n^{3} \quad\left(n^{3}>n^{2}\right)$

Salesman (table[n][3], sCity) $€ \Omega n \quad\left(n<n^{2}\right)$

Salesman (table[n][3], sCity) $€ \Phi n^{2} \quad\left(n^{2}=n^{2}\right)$

Performance wise, it requires 17 seconds for a problem with 100 cities

$(100 !=9.3326215443944152681699238856267 e+157$ permutations)

\section{E. Implementation}

Figure 6 depicts the screenshot of the program that implements the Traveling Salesman problem using C\#.NET.

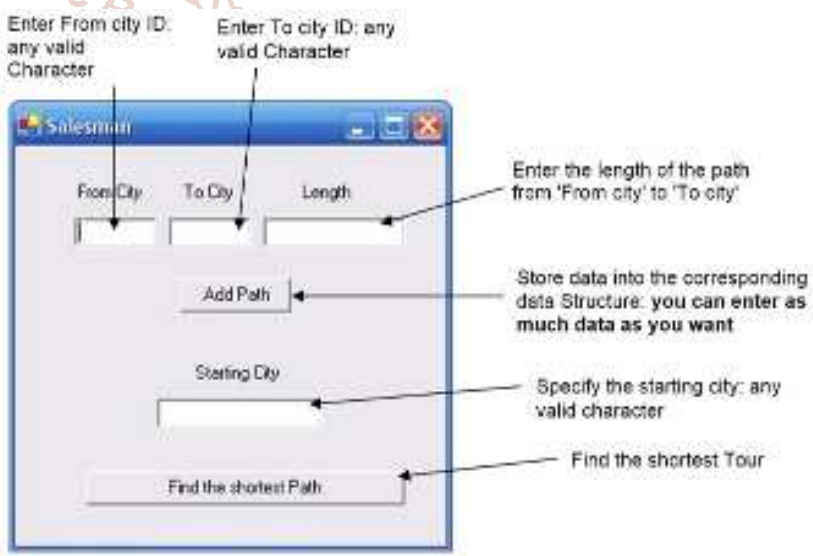

Figure 6: The Traveling Salesman Program

\section{Conclusions \& Future Work}

This paper proposed three new optimized algorithms for solving three combinatorial optimization problems namely the Knapsack problem, the Job Assignment problem, and the Traveling Salesman problem respectively. Each problem was tackled from a design, analysis, and implementation point of views. The proposed designs showed the optimized versions of the algorithms while listing their complete pseudo code. Furthermore, a thorough time complexity analysis was performed to finally end up implementing the algorithms and testing them using C\#.NET.

As future work, the proposed algorithms are to be parallelized using multithreading and multiprogramming techniques so as to speeding up their execution time and making them more adaptable to large computing architectures.

\section{Acknowledgment}

This research was funded by the Lebanese Association for Computational Sciences (LACSC), 
Beirut, Lebanon, under the "Parallel Programming Algorithms Research Project - PPARP2019”.

\section{References}

[1] Caccetta, L., Kulanoot, A, "Computational Aspects of Hard Knapsack Problems". Nonlinear Analysis, 47 (8): 5547-5558, 2001

[2] Poirriez, Vincent; Yanev, Nicola; Andonov, Rumen, "A hybrid algorithm for the unbounded knapsack problem", Discrete Optimization, 6 (1): 110-124, 2009

[3] Petzold, Charles, "Programming Microsoft Windows with C\#", Microsoft Press. ISBN 07356-1370-2, 2002

[4] Munkres, James, "Algorithms for the Assignment and Transportation Problems", Journal of the
Society for Industrial and Applied Mathematics, 5 (1): 32-38, 1957

[5] Brualdi, Richard A., "Combinatorial matrix classes. Encyclopedia of Mathematics and Its Applications", Cambridge: Cambridge University Press, ISBN 978-0-521-86565-4, 2006

[6] Dershowitz, Nachum, "A simplified loop-free algorithm for generating permutations", Nordisk Tidskr Informations, 15 (2): 158-164, 1957

[7] Cook, William, "In Pursuit of the Traveling Salesman: Mathematics at the Limits of Computation", Princeton University Press, ISBN 9780691152707, 2012

[8] Steinerberger, Stefan, "New Bounds for the Traveling Salesman Constant", Advances in Applied Probability, (47): 27-36, 2015. 